\title{
Automatic Household Electrical Lights Monitoring System
}

\author{
${ }^{1}$ K. Aladeniyi, ${ }^{2}$ J.K. Aponjolosun, ${ }^{3}$ S.B. Eniafe, ${ }^{4}$ S. O. Solanke \\ ${ }^{1}$ Department of Science Laboratory Technology, Rufus Giwa Polytechnic, Owo, Ondo State, Nigeria \\ ${ }^{2}$ Department of Electrical Electronics Engineering, Rufus Giwa Polytechnic, Owo, Ondo State Nigeria. \\ ${ }^{3}$ Department of Physics, University of Ibadan, Ibadan. Oyo State, Nigeria \\ ${ }^{4}$ Department of Information Technology, National Open University of Nigeria, Abuja.
}

\begin{abstract}
The popular 555 IC timer incorporated as time delay circuit and operated with a $5 \mathrm{~V}$ DC source was adapted to design and construct an automatic day and night household Electrical lights monitoring and control circuitry for residences.
\end{abstract}

KEYWORD: 555 IC Timers, Comparator, Potentiometer, Photocell, Signal

Submitted Date 31 May 2013

Accepted Date: 06 June 2013

\section{Introduction}

Survey had shown that people generally do not have an effective and automatic means of monitoring and controlling their electrical lighting units automatically during the day and during night. Such a system that switches on all electrical lighting units during night and switches off the units during the day is not installed in many homes [1].

The marvels of the popular 555 IC timer is adopted to design and construct the monitoring and controlling unit called the Automatic Household Electrical Lights Monitoring System.The system is automatic because of the inherent meansof differentiating between shadow and darkness and between daylight and darkness by means of the incorporated time delay circuits and this makes it different from the popular and ordinary light/dark operated switch [1-4].Hence the sensor (photocell) and the entire circuitry only respond to true darkness and true daylight and not shadow and illuminating sources. The system therefore controls the household electrical lamps and security lighting units by ensuring that these are only switched on during night and switched off during daylight circumventing all natural and artificial interference like stray light and lightning during the night and shadow cast on the sensor.The systemcombines three fields of electronics namely; Optoelectronics, analog electronics and digital electronics.

\section{The Design Procedures AND Operation OF VARIOUS Units}

The block diagram of the system is shown in fig.1

The schematic diagram of the Sensoring and Processing unit is shown in fig. 2. This unit senses both darkness and light and processes their corresponding electrical effects for further processing by other stages. The main sensor is the well-known light dependant resistor (LDR) whose resistance is a function of light intensity.

A Light Dependent Resistor (aka LDR, photoconductor, photocell, or photoresistor.) is a device which has a resistance which varies according to the amount of light falling on its surface, when light falls upon it then the resistance changes. Light dependent resistors or LDRs are often used in circuits where it is necessary to detect

The presence of light, or the ambient level of light, often to create a light triggered switch [1,5].

A typical LDR has a resistance in total darkness of $1 \mathrm{M} \Omega$, and a resistance of a couple of $\mathrm{k} \Omega$ in bright light (1020kOhm@10 lux, 2-4kOhm @ 100 lux). It is not uncommon for the values of resistance of an LDR to be several megohms in darkness and then to fall to a few hundred ohms in bright light [5]. Two of its earliest applications were as part of smoke and fire detection systems and camera light meters. Because cadmium sulphide cells are inexpensive and widely available, LDRs are still used in electronic devices that need light detection capability, such as security alarms, street lamps, and clock radios[6]

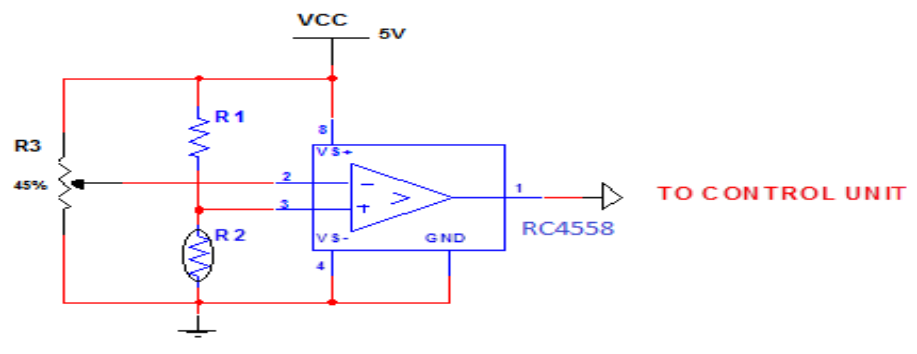

Fig. 2 Sensoring and Processing Un it 
The Op amp $\mathbf{I C}_{\mathbf{1}}(\mathbf{R C 4 5 5 8 )}$ is configured as a comparator.The $\mathbf{R C 4 5 5 8}$ device is a dual generalpurpose operational amplifier, with each half electrically similar to the $\boldsymbol{\mu A \mathbf { A 4 1 }}$. The highcommon-mode input voltage range and the absence of latch-up make this amplifier ideal forvoltage-follower applications. The device is short-circuit protected, and the internal frequency compensation ensures stability without external components [7].

Thepotentiometer $\mathbf{R}_{\mathbf{3}}$ forms a voltage divider whose preset voltage will be compared with the voltage at pin $3\left(\mathrm{~V}_{3}\right)$ at any instant of light intensity. Similarly, $\mathbf{R}_{1}$ and $\mathbf{R}_{\mathbf{2}}$ form a voltage divider, such that the voltage $\mathrm{V}_{3}$ at pin 3at any instant of light intensity changes as the resistance of the Photocell/ $\mathbf{R}_{2}$ changes.

$V_{a}=\frac{V_{c c} \cdot R_{2}}{R_{1}+R_{2}}$

In the daylight, it is known that the resistance of $\mathbf{R}_{2}$ is very LOW,by the action of the comparator and the adjustment of the voltage at pin $2\left(\mathrm{~V}_{2}\right)$ via the potentiometer such that $\mathrm{V}_{3}>\mathrm{V}_{2}$, the output at pin 7 can be made LOW. Hence in daylight, the output of this unit is zero' or a LOW signal.

However at night, the photocell resistance $\mathbf{R}_{2}$ is very high such that $\mathrm{V}_{3}<\mathrm{V}_{2}$ (with previous potentiometeradjustmentintact), the output of this unit is then HIGH.

$10 \mathrm{~K} \Omega$

The following measurements were observed For $\mathrm{V}_{\mathrm{cc}}=6 \mathrm{~V}$, potentiometer preset at $2.7 \mathrm{~K} \Omega$ and $\mathrm{R}_{1}=$

During daylight,

$\mathrm{V}_{2}=4.38 \mathrm{~V}$

$\mathrm{V}_{3}=3.97 \mathrm{~V}$

$\mathrm{V}_{0}=1.38 \mathrm{~V}$

During darkness,

$\mathrm{V}_{2}=3.90 \mathrm{~V}$

$\mathrm{V}_{3}=5.49 \mathrm{~V}$

$\mathrm{V}_{0}=5.80 \mathrm{~V}$

These two output voltage levels are sent to the control unit. These monitor and coordinate the responses of the entire circuitry to variations in daylight and darkness.

The circuit diagram of the control unit is shown in fig3.Transistors $\mathbf{Q}_{\mathbf{1}}$ and $\mathbf{Q}_{\mathbf{2}}$ are switching transistors, $\mathbf{D}_{\mathbf{1}}$ is shadow or night indicator and $\mathbf{D}_{\mathbf{2}}$ is a daylight indicator. $\mathbf{R}_{\mathbf{5}}$ and $\mathbf{R}_{\mathbf{9}}$ are current limiters. $\mathbf{R}_{\mathbf{7}}$ and $\mathbf{R}_{\mathbf{6}}$ form a voltage divider that provides the biasing base current for the switching transistor $\mathbf{Q}_{2}$. $\mathbf{I C}_{2}, \mathbf{R}_{\mathbf{8}}, \mathbf{C}_{\mathbf{1}}$ and $\mathbf{Q}_{2}$ are configured to operate as a directional time delay circuit.

During daylight, when there is zero or LOW voltage signal from the sensoring and processing unit, the transistor $\mathbf{Q}_{1}$ is cut off meaning that $\mathbf{I}_{\mathbf{C}}=0$ and $\mathbf{V}_{\mathbf{C}}$ is $\mathrm{HIGH}$, the general principle of operation of a switching transistor $[8,9,10]$.

The implications of these signals, $\mathbf{I}_{C}$ and $\mathbf{V}_{\mathbf{C}}$ are $\mathbf{D}_{1}$ is OFF and switching transistor $\mathbf{Q}_{2}$ is provided with 'enough positive' base current and hence it is saturated or $\mathbf{Q}_{2}$ is $\mathrm{ON}$. This keeps the trigger input $\mathbf{I C}_{2}$ near ground and the external capacitor $\mathbf{C}_{1}$ is fully discharged. This LOW signal on the trigger input causes the device output to be HIGH. It remains high for the time given as;

$$
t_{d 1}=R_{8} C_{1} \ln 3 \quad \text { [2] }
$$

Thereafter, it goes LOW and hence led $\mathbf{D}_{2}$ glows, indicating daylight.

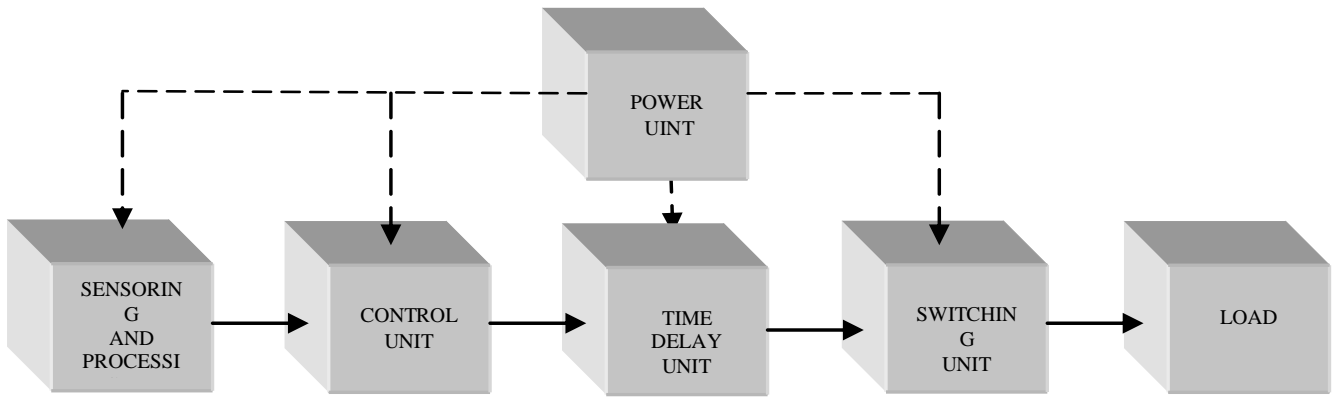

Fig.1the block diagram of the Automatic Household Electrical Lights Monitoring System.

For a $5 \mathrm{~s}$ delay, $\mathrm{R}_{8}=1 \mathrm{M} \Omega, \mathrm{C}_{1}=4.7 \mu \mathrm{F}$. 


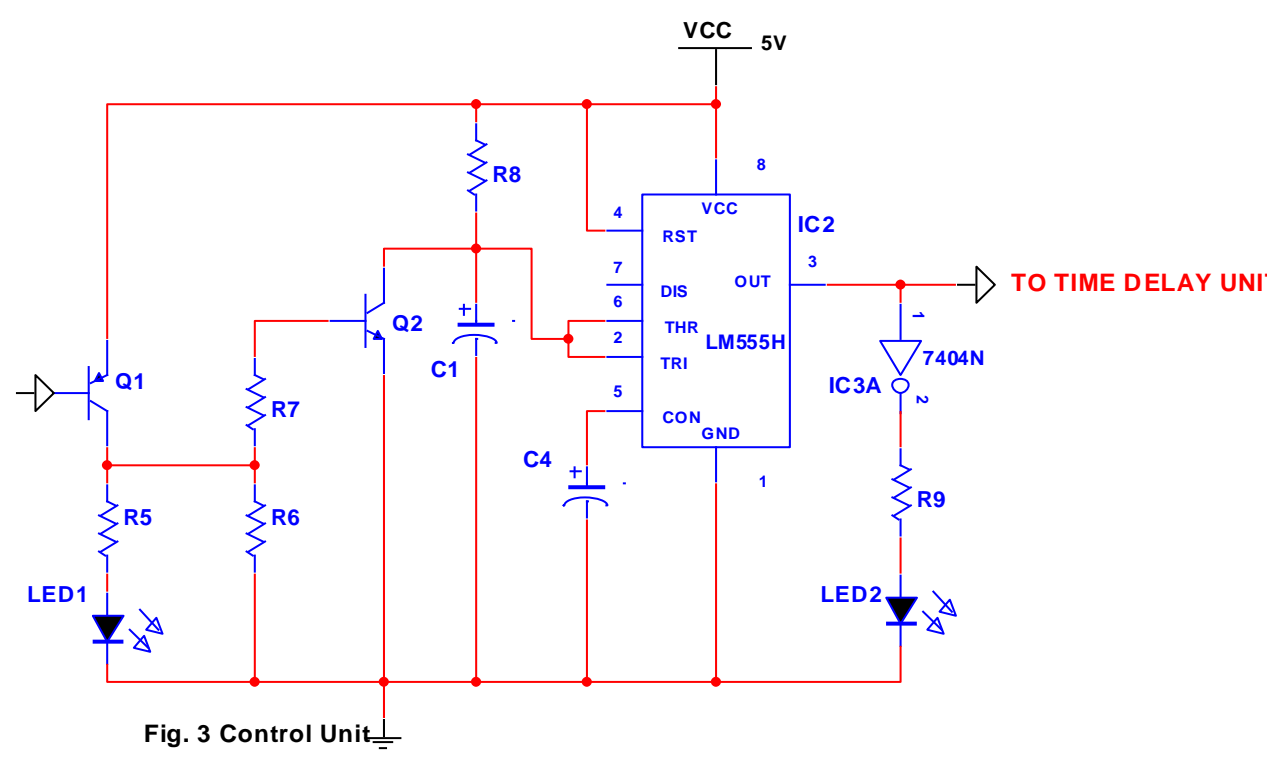

However during darkness, there is HIGH-level signal from the sensoring and processing unit which makes transistor $\mathbf{Q}_{1}$ to be saturated and then $\mathbf{D}_{\mathbf{1}}$ glows. The saturation of $\mathbf{Q}_{\mathbf{1}}$ causes a LOW-level voltage to be applied to the base of $\mathbf{Q}_{2}$ and hence $\mathbf{Q}_{2}$ is cut off. The output does not change correspondingly because $\mathbf{C}_{\mathbf{1}}$ begins to charge through $\mathbf{R}_{\mathbf{8}}$. When the capacitor $\mathbf{C}_{\mathbf{1}}$ voltage reaches ${ }^{2}{ }_{3} \mathbf{V}_{\mathbf{C C}}$, this unit output then switches to the LOW state and remains LOW until $\mathbf{Q}_{2}$ is again saturated. There is therefore a delay in the response of the unit output at pin 3 with respect to the triggering input cause by the switching action of $\mathbf{Q}_{2}$. The essence of incorporating the time delay is to differentiate between darkness and false darkness either due to shadow and changes in weather condition during day. The corresponding waveforms are shown in fig.4.

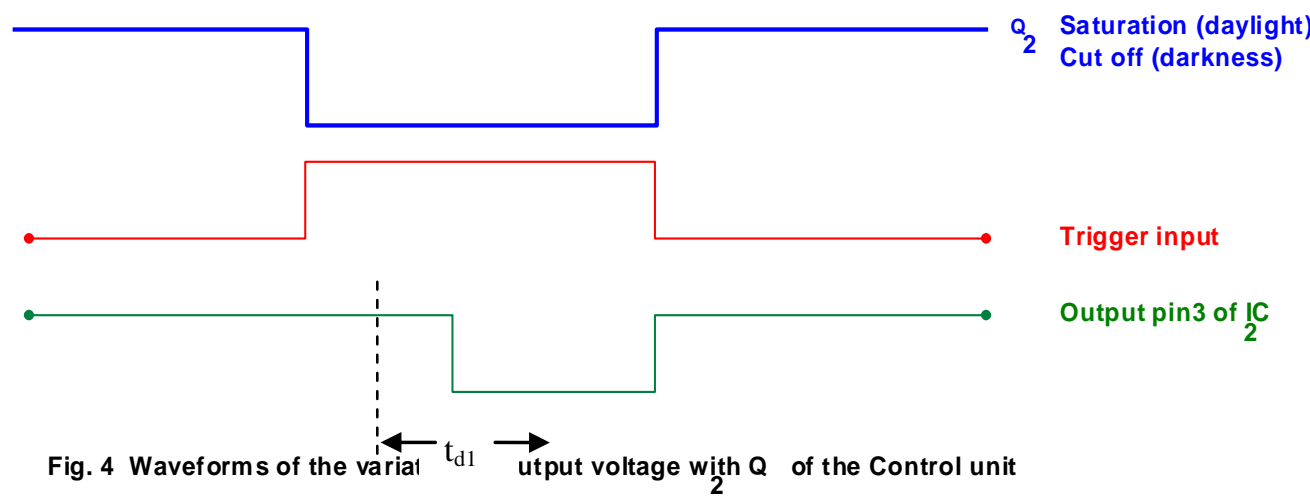

Fig.5 shows schematic diagram of the time delay unit and the switching unit. When the output from the control unit is HIGH i.e. during daylight, transistor $\mathbf{Q}_{3}$ is saturated and capacitor $\mathbf{C}_{2}$ charges towards $\mathbf{V}_{\mathbf{C C}}$ through

$\mathbf{R}_{\mathbf{1 0}}$. It should be noted that $\mathbf{Q}_{3}$ is PNP switching transistor in this case. The output voltage at pin 3 of $\mathbf{I C}_{\mathbf{4}}$ is then at LOW signal, the diode $\mathbf{D}_{3}$ does not glow and thus the Relay is in the OFF position. $\mathbf{Q}_{4}$ serves as a small signal amplifier to provide the quiescent current for the Relay and $\mathbf{R}_{\mathbf{1 2}}$ provides the base bias current for $\mathbf{Q}_{4}$. $\mathbf{R}_{11}$ and $\mathbf{R}_{13}$ are current limiters.

When there is darkness, the output from the control unit changes to LOW after a time delay given byequation 2, the transistor $\mathbf{Q}_{3}$ is then cut off after this time and the output voltage at pin 3 of $\mathbf{I C}_{\mathbf{4}}$ is $\mathrm{HIGH}$. Hence $\mathbf{D}_{\mathbf{3}}$ glows and the Relay is in the 'ON' position. This remains in the 'ON' position until there is daylight. 


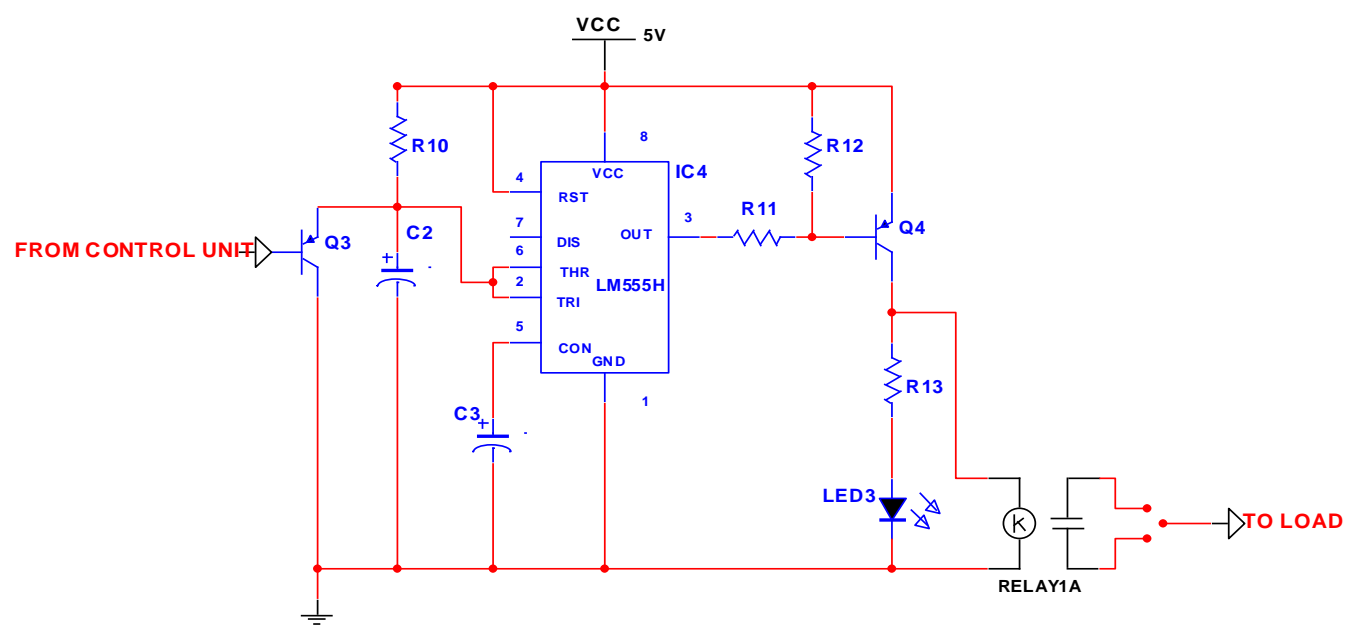

Fig. 5 Schematic diagram of the time delay unit and the switching unit

However, when there is daylight, $\mathbf{Q}_{3}$ will be saturated, but the voltage at pin 3 of $\mathbf{I C}_{4}$ that was initially HIGH does not change correspondingly because $\mathbf{C}_{\mathbf{2}}$ now charges through $\mathbf{R}_{\mathbf{1 0}}$. When the capacitor $\mathbf{C}_{2}$ voltage reaches ${ }^{2} / 3 \mathbf{V}_{\mathbf{C C}}$, the voltage at pin 3 of $\mathbf{I C}_{4}$ then switches to the LOW state. $\mathbf{D}_{3}$ therefore goes OFF and the Relay changes to 'OFF' position. The time delay in the response of the output of $\mathbf{I C}_{\mathbf{4}}$ is given a

$$
t_{d 2}=R_{8} C_{1} \ln 3 \quad[3]
$$

Fig.6 shows the waveforms of the outputs $\mathbf{Q}_{3}$ and $\mathbf{I C}_{4}$ andthe overall effect of $\mathbf{I C}_{3}$ and $\mathbf{I C}_{4}$ is summarized in Fig.7.

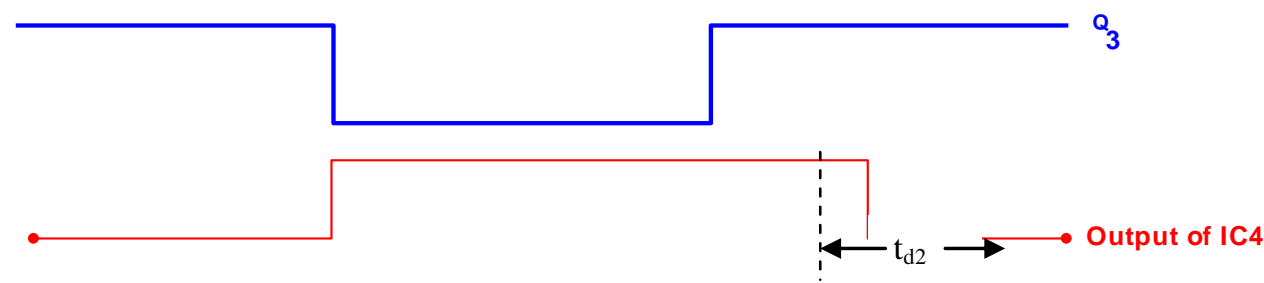

Fig. 6 Waveforms of the outputs of Q3 and IC4

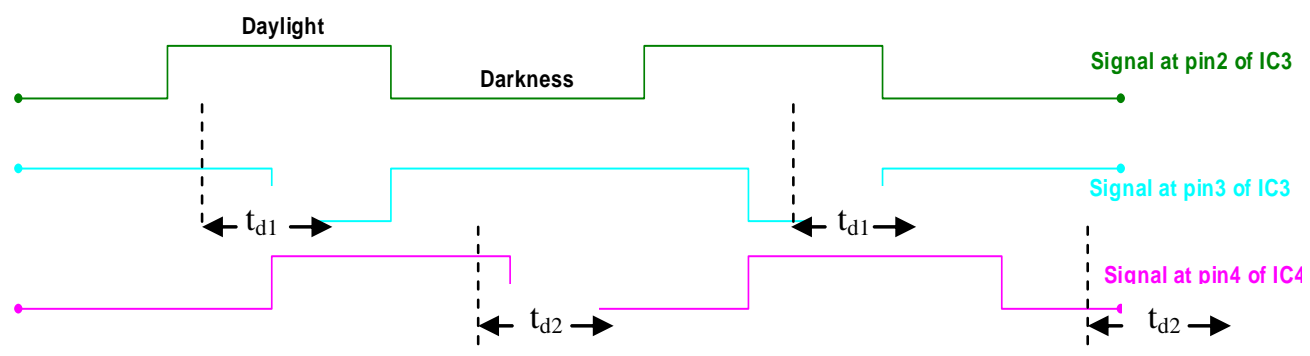

Fig. 7 Waveforms summarizing the flow signals of the system

\section{Conclusion}

This simple project differs from other light/dark operated switch by virtue of its inherent means of differentiating between shadow and darkness and between daylight and darkness by means of the incorporated time delay circuits. Hence the sensor (photocell) and the entire circuitry only respond to true darkness and true daylight and not shadow and illuminating sources. The system therefore controls the household electrical lamps and security lighting units by ensuring that these are only switched on during night and switched off during daylight circumventing all natural and artificial interference like stray light and lightning during the night and shadow cast on the sensor. 


\section{References}

[1]. J. G. Kolo, Development of a Light Dependent Automatic-Off Timer for House Holds Electronics, Leonardo Electronic Journal of Practices and Technologies, 12, 2008,129-138

[2]. W. Jing-Min, L. Chia-Liang,Design and Implementation of a Sun Tracker with a Dual-Axis Single Motor for an Optical SensorBased Photovoltaic System, Sensors, 13, 2013, 3157-3168.

[3]. C. Sarabanan, M. A. Panneerselvam, I. W. Christopher, A Novel Low Automatic Solar Tracking System, International Journal Computer Applications, 31(9), 2011, 62-67.

[4]. Electronics Circuit and Diagram-Electronics Projects and Design retrieved from - http://www.circuitstoday.com/light-activatedswitch-circuit.

[5]. 'LDR' Retrieved fromhttp://www.openobject.org/physicalprogramming/images/archive/c/c9/20100925041628!Sensor_report.pdf

[6]. S. Gajula, S, Badugu, Real Time Adaptable Automation, International Journal of Engineering Trends and Technology, 4(4), 2013, 967-971.

[7]. Dual General-Purpose Operational Amplifier, Retrieved from-http://www.ti.com/lit/ds/symlink/rc4558.pdf

[8]. Electronics Tutorials retrieved from- http://www.electronics-tutorials.ws/transistor/tran 4.html

[9]. V.K., Mekta, R. Mehta, Principle of Electronics( Ram Nagar, New Delhi, S. Chand \& Company LTD, 2004).

[10]. R. J. Tocci, N. S. Widmer, G.L. Moss, Digital System - Principle and application ( Indian, Prentice-Hall, Inc., 2007) 